\title{
Wir wollen Mittelalter!
}

\section{Didaktisches Potential für den Schulgeschichtsunterricht}

Dunkel, rückständig, romantisch, aufregend - vielfältig sind die Vorstellungen vom Mittelalter, geprägt von Burgen und Drachen in deutschen Kinderzimmern. ${ }^{1} \mathrm{Zu}$ keiner anderen Epoche ist das Geschichtsbild von Schülerinnen und Schülern derart vorgefestigt wie zur Zeit der Ritter und Burgfräulein. Allen gemein ist die Überzeugung, dass das Mittelalter losgelöst sei von der Gegenwart, eine fremde und untergegangene Welt, deren Existenz zwar fasziniert, jedoch ohne jeden Einfluss auf die eigene Lebenswirklichkeit bleibt. Wer will sich angesichts dieser vermeintlichen Tatsache schon ernsthaft mit dieser Zeit im Schulgeschichtsunterricht auseinandersetzen oder, überspitzt formuliert, wer will schon Mittelalter? Es sind jedoch gerade die scheinbar festgefahrenen Geschichtsbilder, die vielversprechende Anknüpfungspunkte für einen modernen Unterricht bieten, der die Bedeutung der Epoche im Sinne einer gemeinsamen Erinnerungskultur, des kollektiven Gedächtnisses und einer gemeinsamen Identität erschließen kann. Für den Schulgeschichtsunterricht eröffnet die Beschäftigung mit dem Mittelalter vielfältige didaktische Möglichkeiten, auf die es sich lohnt, einen näheren Blick zu werfen.

\section{Mittelalter und Populärkultur}

Längst hat das Mittelalter Eingang gefunden in die Populärkultur. Ob Könige, Ritter oder Wikinger - sie begegnen uns in Spielzeug, populärwissenschaftlichen Zeitschriften und Büchern, in Videospielen und Filmen. ${ }^{2}$ Entstanden ist eine Subkultur von beträchtlicher ökonomischer Bedeutung. Treffend hat Fabian Link bemerkt: „Mittelalter ist ein Verkaufsschlager [...].“3 Dabei ergeben sich aus der Tatsache, dass Geschichte nicht mehr nur über historische Institutionen tradiert wird, divergierende Geschichtsbilder zwischen formaler, non-formaler und informeller Bildung. Über die im Handel angebotenen Produkte erfolgt ein Spiel mit den Erwartungen des Käufers an das Mittelalter.

\footnotetext{
1 Zu den verschiedenen Geschichtsbildern siehe Meike Hensel-Grobe, Divergierende Geschichtsbilder? Das (Spät-) Mittelalter im Dialog zwischen Universität und Geschichtsunterricht, in: Regina Toepfer (Hrsg.), Das Mittelalter. Perspektiven mediävistischer Forschung. Zeitschrift des Mediävistenverbandes, 22, 2017, 1. 75-92, hier 76.

2 Simon Maria Hassemer, Das Mittelalter der Populärkultur. Medien - Designs - Mytheme. Freiburg i.Br. 2014.

3 Fabian Link, Gegenwarten des Mittelalters vom späten 18. bis ins 20. Jahrhundert. Politisierung, Populärkultur und die Kulturwissenschaften, in: Geschichte in Wissenschaft und Unterricht (GWU) 9/10, 2016, 509-522, hier 509.

>> Das Werk ist unter der Creative-Commons-Lizenz Namensnennung - Weitergabe unter gleichen Bedingungen 4.0 International veröffentlicht. Den Vertragstext finden Sie unter: https://creativecommons.org/licenses/by-sa/4.0/deed.de. Bitte beachten Sie, dass einzelne, entsprechend gekennzeichnete Teile des Werks von der genannten Lizenz ausgenommen sein bzw. anderen urheberrechtlichen Bedingungen unterliegen können.
} 
Aus dieser Erkenntnis heraus entsteht auch bei Schülerinnen und Schülern ein von der Populärkultur geformtes und zugleich auf sich selbst rekurrierendes Geschichtsbild, das mit den Ergebnissen der Mittelalterforschung kaum etwas gemein hat. ${ }^{4}$

Gerade mit dem von der Populärkultur geformten Mittelalterbild eröffnen sich didaktische Möglichkeiten für den Schulgeschichtsunterricht. Meike Hensel-Grobe hat bemerkt, die unter Schülerinnen und Schülern verbreiteten Geschichtsbilder zum Mittelalter tendierten zwischen „romantisch-glanzvoll“ und „finster-rückständig“, zudem würden sie als „Negativfolie für die Betrachtung von modernen Gesellschaften“ herangezogen. ${ }^{5}$ Das scheinbar abenteuerlich-romantische Leben der Ritter werde in der Vorstellungwelt der Schülerinnen und Schüler zugleich mit sozialer Ungleichheit, hoher Gewaltbereitschaft, einer statischen Gesellschaftsordnung und primitiven Lebensweise verbunden. ${ }^{6}$ Die Erfahrungen im täglichen Schulunterricht verdeutlichen, dass sich derartige - nicht zuletzt aus der Populärkultur hervorgegangene - Überzeugungen der Schülerinnen und Schüler von der Überlegenheit der eigenen Gegenwartsgesellschaft gegenüber früheren Jahrhunderten keineswegs auf die Zeit des Mittelalters beschränken. In der eigenen Unterrichtspraxis stößt man immer wieder seitens der Lernenden auf Unverständnis gegenüber den Menschen der Altsteinzeit und ihrem Leben als Nomaden oder den Wählerinnen und Wählern Hitlers, die doch vermeintlich hätten erkennen müssen, welche Folgen die Wahl der NSDAP mit sich bringen werde. Indem derartige Vorstellungen der Schülerinnen und Schüler bereits in den unteren Jahrgangsstufen der weiterführenden Schulen aufgebrochen werden, kann historisches Denken entwickelt, Multiperspektivität vermittelt werden und Fremdverstehen gelingen. Das Mittelalter mit seinen scheinbar etablierten Geschichtsbildern bietet sich hier in besonderer Weise an.

\section{Mittelalter als Alteritäts- und Similaritätserfahrung}

In der Beschäftigung mit der Alterität des Mittelalters liegt das besondere Potential für den Schulgeschichtsunterricht der Sekundarstufe I. Indem die Andersartigkeit der mittelalterlichen Gesellschaft und des Alltagslebens nicht aus einer überheblichen und scheinbar überlegenen Perspektive der Gegenwart heraus betrachtet, sondern wertneutral als Bedingung jeder Beschäftigung mit der Vergangenheit anerkannt wird, können den Schülerinnen und Schülern wesentliche Grundprinzipien der Geschichte begreiflich gemacht werden. Dabei sind die in den Köpfen zunächst vorhandenen Stereotype keineswegs, wie Bernhard Jussen gefordert hat, Anlass, den Begriff ,Mittelalter` nicht mehr

\footnotetext{
4 Zur Konstruktion von Mittelalterbildern im Sinne des Medievalism und Neomedievalism siehe Hassemer, Das Mittelalter der Populärkultur (wie Anm. 2), 19-42.

5 Hensel-Grobe, Divergierende Geschichtsbilder (wie Anm. 1), 76.

6 Ebd., 82.
} 
zu verwenden. ${ }^{7}$ Vielmehr können diese Stereotype, Klischees und Vorurteile selber zum Unterrichtsgegenstand gemacht werden, so dass die Schülerinnen und Schüler nicht nur inhaltlich etwas über diese Epoche lernen, sondern befähigt werden, reflektierte Werturteile im Sinne der Orientierungskompetenz über den eigenen Umgang mit der Vergangenheit zu fällen.

Für den Geschichtsunterricht der Sekundarstufe II birgt weniger die Alterität als vielmehr die Similarität von Mittelalter und Gegenwart ein weitreichendes didaktisches Potential. Verständlich erscheint Bernhard Jussens Kritik an einer ausschließlich auf die Andersartigkeit des Mittelalters ausgerichteten Unterrichtspraxis, wenn er bemerkt: „Wer das Wort ,Mittelalter` benutzt, baut einen pawlowschen ,Kontrastbewußtstein“Auslöser in seine historische Darstellung ein. ${ }^{\text {"8 }}$ Die Epoche soll von Schülerinnen und Schülern eben nicht als "Negativfolie“ begriffen werden, sondern als dynamische Zeit, aus der wesentliche Strukturen der Gegenwart entwuchsen. Um Entwicklungen, die sich über Jahrhunderte erstreckten, erfassen zu können, erscheint es notwendig, die Chronologie des Geschichtsunterrichts aufzubrechen, wie dies beispielsweise für die Oberstufe in Schleswig-Holstein vorgesehen ist. Das im ersten Lernjahr der Sekundarstufe II z. B. in Schleswig-Holstein zu unterrichtende Thema „Wandel von Wirtschaft und Gesellschaft Kontinuitäten und Brüche" schlägt einen Bogen vom Feudalismus bis in die globalisierte Gegenwart. ${ }^{9}$ Soziale Veränderungen vom Gesellschaftssystem des Mittelalters über das Zeitalter der Industrialisierung bis zu heutigen Gesellschaftsformen sollen den Schülerinnen und Schülern vermittelt werden. Dazu bietet es sich an, wenngleich kein Produkt der neuesten Forschung, auf das Modell der ,langen Dauer (la longue durée) von Fernand Braudel zurückzugreifen. Bereits Ende der 1950er Jahre hat der Vertreter der AnnalesSchule die überwiegende Beschäftigung der Geschichtsforschung mit der chronologischen Abfolge von Einzelereignissen angeprangert und dafür plädiert, Entwicklungen in größeren zeitlichen Zusammenhängen zum Untersuchungsgegenstand zu machen. Dazu unterscheidet er historische Einzelereignisse (l'histoire événementielle) von konjunkturellen Entwicklungen (la moyenne durée) und sich langsam entwickelnden Strukturen (la longue durée), die vor allem, doch nicht nur in der Sozialgeschichte von Bedeutung sind. ${ }^{10}$ Dabei betont auch er, dass die Gegenwart nur unter Berücksichtigung der Alterität der Vergangenheit verstanden werden könne, die Geschichte mithin kein Selbstweck sei: „Hüten wir uns vor dem l'art pour l'art.“11 Damit entspricht Braudel der Auffassung auch

7 Bernhard Jussen, Richtig denken im falschen Rahmen? Warum das, Mittelalter' nicht in den Lehrplan gehört, in: Geschichte in Wissenschaft und Unterricht (GWU) 9/10, 2016, 558-576, hier 561.

8 Ebd., 569.

9 Ministerium für Schule und Berufsbildung des Landes Schleswig-Holstein (Hrsg.), Fachanforderungen Geschichte. Allgemein bildende Schulen, Sekundarstufe I, Sekundarstufe II. Kiel 2016, 29, https://www.schulpraxis-paedagogik.uni-kiel.de/de/ dateien/fachanforderungen/fa-gesch (letzter Zugriff: 26.5.2021).

10 Fernand Braudel, Die lange Dauer [La longue durée], in: Theodor Schieder/Kurt Gräubig (Hrsg.), Theorieprobleme der Geschichtswissenschaft. Darmstadt 1977, 164-204.

11 Ebd., 182. 
der modernen Geschichtsdidaktik, die nicht auf der Vermittlung von Einzelereignissen im Sinne der ,histoire événementielle beruht, sondern auf strukturellen Zusammenhängen und zur eigenen Urteilsbildung befähigen soll.

\section{Mittelalter und sozialer Wandel}

Die Beschäftigung mit sozialen Veränderungen vom Mittelalter bis in die Neuzeit bietet sich im Geschichtsunterricht der Sekundarstufe II an, um im Sinne Braudels Veränderungen der longue durée zu erfassen und zu erkennen, dass Strukturen der Gegenwart historisch gewachsen und auf ein keineswegs statisches, sondern äußerst dynamisches Mittelalter zurückzuführen sind. Vom Gesellschaftssystem des Feudalismus kann, trotz sicherlich berechtigter Kritik an der meist als zu absolut dargestellten Lehnspyramide, über die Entwicklung der mittelalterlichen Stadt und des Frühkapitalismus eine Verbindung hergestellt werden zu modernen Wirtschafts- und Gesellschaftsformen. ${ }^{12}$ Dabei soll nicht im marxistischen Sinne, auch wenn Braudel selber auf Marx' Geschichtsvorstellungen als „echte soziale Modelle“ im Sinne der longue durée verwiesen hat, die soziale Entwicklung als Auseinandersetzung zwischen „Oben und Unten“, „Reich und Arm“ oder „Bourgeoisie“ und „Proletariat“ verstanden werden. ${ }^{13}$ Wohl bietet aber der Zugang über das Modell der longue durée den Schülerinnen und Schülern die Möglichkeit, die marxistische Theorie vom ewigen „Klassenkampf“ zu beurteilen und nicht zuletzt auf heutige Strukturen zu beziehen. Auf diese Weise werden die Schülerinnen und Schüler nicht nur die Alterität des Mittelalters und soziale Umbrüche erkennen, sondern auch die Gemeinsamkeiten sozialer Beziehungsgeflechte. Dabei können die von Meike Hensel-Grobe konstatierten Vorstellungen des Mittelalters als „romantisch-glanzvoll“ oder „finster-rückständig“ verworfen und die Epoche als Grundstein für unsere heutige Gesellschaft erfasst werden.

\section{Mittelalter und Erinnerungskultur}

Eine wesentliche Bedeutung kommt der Epoche des Mittelalters im Schulgeschichtsunterricht nicht nur in der Beschäftigung mit Alterität und Similarität zu, sondern auch im Sinne einer gemeinsamen Erinnerungskultur. Bislang fußt die Forschung zur kulturellen Identität vor allem auf den Erfahrungen des Nationalsozialismus, die als Negativfolie herangezogen werden. So rekurrieren Studien zum kollektiven Gedächtnis und der gemeinsamen Erinnerungskultur meist auf die Zeit zwischen 1933 und 1945. Entspre-

12 Zur Kritik an der Vermittlung von Lehnspyramide und Lehnswesen im Schulgeschichtsunterricht siehe Arnold Bühler, Keine Lehnspyramide! Kein Lehnswesen! Plädoyer für eine Entrümpelung des Mittelalter-Unterrichts, in: Geschichte in Wissenschaft und Unterricht (GWU) 3/4, 2019, 136-148, hier 148.

13 Zu Braudels Ausführungen zur marxistischen Theorie siehe Braudel, Die lange Dauer (wie Anm. 10), 202. 
chend hat Astrid Erll bemerkt, dass im kollektiven Gedächtnis der Holocaust Ereignisse wie beispielsweise den Völkermord an den Herero und Nama gänzlich überlagere. ${ }^{14}$ Auch Jan und Aleida Assmann haben auf die Bedeutung des Nationalsozialismus für das kollektive Gedächtnis hingewiesen. ${ }^{15}$ Christoph Cornelißen hat betont, dass, vor allem politisch motiviert, versucht werde, gesamteuropäische Erinnerungsorte zu schaffen, und die Befreiung des Vernichtungslagers Auschwitz als Beispiel genannt. ${ }^{16}$ Für die sich vor tausend Jahren zugetragenen Ereignisse scheint demgegenüber kein Platz im kollektiven Gedächtnis zu verbleiben.

Doch auch die Epoche des Mittelalters war in der Vergangenheit und ist zum Teil bis in die Gegenwart hinein Kristallisationspunkt einer gemeinsamen Erinnerungskultur, eines kollektiven Gedächtnisses. Zurecht hat Fabian Link bemerkt, das Mittelalter sei „[...] seit seinem künstlich festgelegten Ende für die jeweiligen Gegenwarten neu verwendet und instrumentalisiert worden. ${ }^{\text {"17 }}$ Er verweist dabei auf die Zeit des Nationalsozialismus, für die das Mittelalter zur Legitimation des eigenen völkisch-rassischen Gesellschaftsmodells und Herrscherfiguren wie Heinrich der Löwe im Zuge der Ostsiedlung zum Vorreiter der Eroberung von „Lebensraum im Osten“ stilisiert wurden. ${ }^{18}$ Dem entsprechen die Ergebnisse einer Untersuchung von Schulbüchern aus der Zeit des ,Dritten Reichs', der DDR und der frühen Bundesrepublik von Martin Clauss und Martin Munke. Die Autoren haben angemerkt, dass die Ostsiedlung durch den Nationalsozialismus im Sinne der „Blut-und-Boden-Ideologie“ interpretiert und der Antisemitismus historisch legitimiert worden sei. ${ }^{19}$ Im Schulbuch der DDR werde wiederum der Begriff des Mittelalters durch den des ,Feudalzeitalters' ersetzt und damit bereits eine inhaltliche Zielsetzung deutlich, die die Epoche als Klassenkampf ausgebeuteter Bauern gegen ihre Feudalherren darstellt. ${ }^{20}$ Über eine Untersuchung solch verschiedener Mittelalterbilder können heutige Schülerinnen und Schüler erkennen, welche Bedeutung der Geschichte als politisches Instrument zur Legitimation eigener Herrschaftsansprüche zukommen kann und inwiefern sie als Mittel dient, um Identität im Sinne einer gemeinsamen Erinnerungskultur zu stiften.

Die Geschichte des Mittelalters als identitätsbildendes Element erschöpft sich nicht in den Narrationen des Nationalsozialismus oder der DDR. Der Mythos Karls des Gro-

\footnotetext{
14 Astrid Erll, Kollektives Gedächtnis und Erinnerungskulturen. Eine Einführung, 3. Aufl. Stuttgart 2017, 120.

15 In ihrer Dankesrede zur Verleihung des Friedenspreises des Deutschen Buchhandels 2018 haben Jan und Aleida Assmann betont, dass Erinnerung an die beschämende Geschichte identitätsstiftend für eine Gesellschaft sein könne. Siehe Aleida Assmann/Jan Assmann, Dankesrede, in: O.V., Friedenspreis des Deutschen Buchhandels 2018. Ansprachen aus Anlass der Verleihung. Frankfurt a. M. 2018, 53-65, hier 59f.

16 Christoph Cornelißen, Die Nationalität von Erinnerungskulturen als ein gesamteuropäisches Phänomen, in: Geschichte in Wissenschaft und Unterricht (GWU) 62, 2011, 5-16, hier 14.

17 Link, Gegenwarten des Mittelalters (wie Anm. 3), 509.

18 Ebd., 510 und 520 .

19 Martin Clauss/Martin Munke, Mittelalter-Bilder im Schulbuch. NS-Deutschland, Bundesrepublik und DDR im Vergleich, in: Geschichte in Wissenschaft und Unterricht (GWU) 9/10, 2016, 577-587, hier 580f.

20 Siehe Ebd., 584.
} 
ßen als „Vater Europas“, der zuletzt von Alessandro Barbero publikumswirksam bemüht wurde, verfolgt nicht zuletzt das Ziel der Schaffung eines supranationalen europäischen Bewusstseins und erweist sich damit ebenfalls als Instrument politischer Ziele in der Gegenwart. ${ }^{21}$ Schülerinnen und Schülern den Konstruktcharakter von Geschichte zu vermitteln, diese als Produkt von Interpretation in Abhängigkeit von der jeweiligen Gegenwart und als Spielball politischer Beeinflussung, von Ideologien und Weltanschauungen begreifbar zu machen, ist zentrales Ziel des Schulgeschichtsunterrichts. Die Beschäftigung mit der Epoche des Mittelalters kann dazu beitragen, dieses Ziel zu erreichen.

\section{Autor}

\section{Sven Hamann}

Gymnasiallehrer und Lehrbeauftragter im Bereich Didaktik der Geschichte an der Christian-Albrechts-Universität zu Kiel. Seine Schwerpunkte in Forschung und Lehre sind der Nationalsozialismus sowie Museumsdidaktik und Erinnerungskultur.

hamann@histosem.uni-kiel.de

21 Alessandro Barbero, Karl der Große. Vater Europas. Stuttgart 2007. 